\title{
LOCAL COHOMOLOGY OF LOGARITHMIC FORMS
}

\author{
GRAHAM DENHAM, HAL SCHENCK, MATHIAS SCHULZE, MAX WAKEFIELD, \\ AND ULI WALTHER
}

\begin{abstract}
Let $Y$ be a divisor on a smooth algebraic variety $X$. We investigate the geometry of the Jacobian scheme of $Y$, homological invariants derived from logarithmic differential forms along $Y$, and their relationship with the property that $Y$ be a free divisor.

We consider arrangements of hyperplanes as a source of examples and counterexamples. In particular, we make a complete calculation of the local cohomology of logarithmic forms of generic hyperplane arrangements.
\end{abstract}

\section{Contents}

1. Introduction

1.1. Logarithmic forms and vector fields

1.2. Euler homogeneity and Jacobians

1.3. Arrangements

2. Embedded primes of the Jacobian ideal

2.1. The algebraic case

2.2. Arrangements

2.3. Analytic divisors

3. Homological properties of logarithmic forms 11

3.1. Higher forms as exterior products 11

4. Generic arrangements 14

Acknowledgments 19

References 19

\section{INTRODUCTION}

Positioned in the singularity hierarchy somewhat opposite to a isolated singularity, a free divisor is a reduced hypersurface with large but well-behaved singular locus. More precisely, freeness is equivalent to the scheme defined by the Jacobian ideal being empty or Cohen-Macaulay of minimal possible codimension. Free divisors occur naturally in deformation theory, as discriminants in base spaces of versal

Date: October 27, 2018.

1991 Mathematics Subject Classification. 32S22, 52C35, 16W25.

Key words and phrases. hyperplane, arrangement, logarithmic, differential form, free.

G.D. supported by NSERC.

H.S. supported by NSA H98230-11-1-0170, NSF DMS-1068754.

M.W. has been partially supported by the Office of Naval Research.

U.W. supported by NSF grant DMS-0901123. 
deformations. The classical case, studied by K. Saito [27], is that of a versal deformation of an isolated singularity. Later, Looijenga [18] expanded Saito's ideas to isolated complete intersection singularities; following Bruce [3] and Zakalyukin [38], Looijenga's results [18, Cor. 6.13] prove freeness of discriminants of stable mappings $f:\left(\mathbb{C}^{n}, 0\right) \longrightarrow\left(\mathbb{C}^{p}, 0\right), n \geq p$. For versal deformations of space curves in 3 -space, van Straten [34] proved freeness of the reduced discriminant. Terao [33] showed that the discriminant of any finite map is free. Apart from discriminants, there are other natural sources of free divisors in this setup, see for instance [21].

Via deep results of Brieskorn [2] and Slodowy [30], free divisors are linked to representation theory: the discriminants of ADE singularities are discriminants of finite Coxeter groups with the same name. It turns out that all Coxeter arrangements (even unitary reflection arrangements) as well as their discriminants are free (see [32]). This led to the study of general free arrangements, and finally to Terao's conjecture, stating that freeness of an arrangement is a combinatorial property. It is one of the most prominent open conjectures in the field, and motivated the results in this article.

While reflection groups are discrete, more recently also free divisors associated with (reductive) algebraic groups have been studied (see [13, 12, 28, 8, 15]). For example, the free divisors associated with a semisimple group are exactly the free discriminants in Sato-Kimura's classification of irreducible prehomogeneous vector spaces, and there are exactly four of them up to castling transformations.

There is also a purely ring theoretic version of Saito's theory of free divisors due to Simis [29].

In this article, we study homological invariants that stand in the way of freeness. Our motivation comes from the study of hyperplane arrangements, but many results are true for more general divisors.

1.1. Logarithmic forms and vector fields. Throughout this paper $X$ will be an $\ell$-dimensional smooth algebraic variety over an algebraically closed field $\mathbb{K}$. We shall also be concerned with affine $\ell$-space

$$
V=\mathbb{A}_{\mathrm{K}}^{\ell}
$$

over an arbitrary field $\mathbb{K}$. In both cases, the sheaf $\Omega_{X}^{1}$ of differentials on $X$ is locally free. By $\mathscr{O}=\mathscr{O}_{X}$ we denote the sheaf of regular functions on $X$. We shall freely identify any quasi-coherent sheaf on an affine scheme with its module of global sections. In case $X=V$, we pick coordinates $\boldsymbol{x}=x_{1}, \ldots, x_{\ell}$ on $V$, and denote by

$$
S=\mathbb{K}[V]=\mathbb{K}[\boldsymbol{x}]
$$

the coordinate ring of $V$. We shall reserve the symbol $R$ to denote arbitrary regular rings.

In general, choosing a regular system of parameters $c_{1}, \ldots, c_{\ell}$ near $x \in X$ induces an étale map $c: U \longrightarrow \mathbb{A}_{\mathrm{K}}^{\ell}$ from any open set $U$ on which the differentials of the $c_{i}$ are linearly independent (see for example [1, VI.1.3]). By construction, $\boldsymbol{c}$ is the pullback of $\boldsymbol{x}$ on $\mathbb{A}_{\mathrm{K}}^{\ell}$ to $U$, and we call it a local coordinate system on $U \subset X$ near $X$; since the map $\boldsymbol{c}$ is étale, the partial differentiation operators $\partial_{i}$ on $\mathbb{A}_{\mathrm{K}}^{\ell}$ lift to vector fields $\eta_{i}$ near $x$ that satisfy $\left[\eta_{i}, c_{j}\right]=\delta_{i, j}$. On an analytic (but generally not on any algebraic) small neighborhood of $x, \boldsymbol{c}$ can be made injective. When we choose $x \in X$ and suitable $U, \boldsymbol{c}, \eta$ in the above sense, we shall abuse notation and denote $\eta$ by $\partial$ and $\boldsymbol{c}$ by $\boldsymbol{x}$. 
Let $\operatorname{Der}_{X}$ be the locally free $\mathscr{O}$-module of vector fields on $X$; for $X=V$, its global sections form the module of $\mathbb{K}$-linear derivations $\bigoplus_{i=1}^{\ell} S \cdot \frac{\partial}{\partial x_{i}}$ on $S$. Let $\Omega_{X}^{\bullet}$ be the algebraic de Rham complex on $X$; if $X=V$, then $\Omega_{X}^{1}$ is the module with global sections $\bigoplus_{i=1}^{\ell} S \mathrm{~d} x_{i}$. In general, $\Omega_{X}^{p}=\bigwedge^{p} \Omega_{X}^{1}$ is a locally free $\mathscr{O}_{X}$-module but the maps in $\Omega_{X}^{\bullet}$ are not $\mathscr{O}_{X}$-linear.

Let

$$
\iota: Y \hookrightarrow X
$$

be a reduced divisor on $X$ and denote by $f$ a reduced local defining equation. Recall that $\mathscr{O}_{X}(k Y)$ is the locally free rank-1 $\mathscr{O}_{X}$-module, locally defined as $\frac{1}{f^{k}} \mathscr{O}_{X}$. With $\mathscr{O}_{X}(Y)=\mathscr{O}_{X}(1 Y)$, for any sheaf $\mathscr{F}$ on $X$, let $\mathscr{F}(Y)=\mathscr{F} \otimes_{\mathscr{O}} \mathscr{O}_{X}(Y)$. On the other hand $\mathscr{O}_{X}(* Y)$ is by definition $j_{*} \mathscr{O}_{X \backslash Y}$, where $j: X-Y \longrightarrow X$ is the open embedding. Our main focus is on the following two objects derived from $Y$ :

Definition 1.1. The sheaf of logarithmic vector fields along $Y$ is the $\mathscr{O}$-module

$$
\operatorname{Der}_{X}(-\log Y)=\left\{\theta \in \operatorname{Der}_{X} \mid \theta(\mathscr{O}(-Y)) \subseteq \mathscr{O}(-Y)\right\} .
$$

The $\mathscr{O}$-module complex $\Omega_{X}^{\bullet}(\log Y)$ of logarithmic differential forms along $Y$ is the largest subcomplex of $\Omega_{X}^{\bullet}(* Y)$ contained in $\Omega_{X}^{\bullet}(Y)$ :

$$
\Omega_{X}^{p}(\log Y)=\left\{\omega \in \Omega^{p}(Y) \mid \mathrm{d} \omega \in \Omega^{p+1}(Y)\right\} .
$$

Geometrically, vector fields in $\operatorname{Der}(-\log Y)$ are tangent to the smooth locus of $Y$.

Convention 1.2. If $X$ is understood from the context, we suppress it in the subscripts in $\mathscr{O}_{X}, \Omega_{X}^{p}, \operatorname{Der}_{X}(-\log Y)$ and $\Omega_{X}^{p}(\log Y)$.

Obviously, $\Omega^{p}(\log Y) \neq 0$ only for $0 \leq p \leq \ell$, and one has $\Omega^{0}(\log Y)=\mathscr{O}$ and $\Omega^{\ell}(\log Y)=\Omega^{\ell}(Y)$. If $X=V$ then $\Omega^{\ell}(\log Y)=\frac{1}{f} S \mathrm{~d} \boldsymbol{x}$ where $\mathrm{d} \boldsymbol{x}=\mathrm{d} x_{1} \wedge \ldots \wedge \mathrm{d} x_{\ell}$.

In [9], an alternative definition is used for $\operatorname{Der}(-\log Y)$ and $\Omega_{V}^{\bullet}(\log Y)$. Our definition agrees with theirs in the arrangement case, but works more generally. By $[9, \S 2], \operatorname{Der}(-\log Y)$ and $\Omega_{V}^{\bullet}(\log Y)$ are reflexive and hence $Y$-normal in the sense of [9]. This implies that certain properties of $Y$, obviously valid at all smooth points, are retained at the singular points of $Y$. For instance:

(1) $\Omega^{\bullet}(\log Y)$ is stable under contraction against elements of $\operatorname{Der}(-\log Y)$.

(2) Contraction sets up a perfect pairing

$$
\operatorname{Der}(-\log Y) \times \Omega^{1}(\log Y) \longrightarrow \mathscr{O}(Y)
$$

and an identification

$$
\operatorname{Der}(-\log Y)=\Omega^{\ell-1}(\log Y)
$$

for $X=V$, these are defined by $\left(\sum_{i} \eta_{i} \mathrm{~d} x_{i}, \sum_{i} \theta_{i} \frac{\partial}{\partial x_{i}}\right) \mapsto \sum_{i} \theta_{i} \eta_{i}$ and $\sum_{i} \eta_{i} \frac{\partial}{\partial x_{i}} \leftrightarrow \sum_{i}(-1)^{i} \frac{\eta_{i}}{f} \mathrm{~d} x_{1} \wedge \ldots \wedge \widehat{\mathrm{d} x_{i}} \wedge \ldots \wedge \mathrm{d} x_{\ell}$ respectively.

(3) The exterior product induces a perfect pairing

$$
\Omega^{p}(\log Y) \times \Omega^{\ell-p}(\log Y) \longrightarrow \Omega^{\ell}(\log Y) .
$$

(4) The natural map $j_{p}: \bigwedge^{p} \Omega^{1}(\log Y) \longrightarrow \Omega^{p}(\log Y)$ is injective.

Definition 1.3. A free divisor is a divisor $Y$ for which $\Omega^{1}(\log Y)$ is a free module. As it turns out, for free $Y$, the modules $\operatorname{Der}(-\log Y)$ and $\Omega^{p}(\log Y), 0 \leq p \leq \ell$, are all free as well and $\Omega^{p}(\log Y)=\bigwedge^{p} \Omega^{1}(\log Y)$. The free locus of the divisor $Y$ is the set of points $x \in Y$ where $\Omega^{1}(\log Y)$ is a locally free $\mathscr{O}$-module; it includes the complement of the singular locus of $Y$ in $X$. 
1.2. Euler homogeneity and Jacobians. Let $U \subseteq X$ be an affine open subset and choose a local defining equation $f$, defined up to units, of $U \cap Y$ relative to some local coordinate system $\boldsymbol{x}$ on $U$. While the particular choice of the coordinate system is relevant, we hide its presence in the symbol " $U$ ".

We denote

$$
\mathscr{J}_{U, f}=\mathscr{O}_{U}\left\langle\frac{\partial f}{\partial x_{1}}, \ldots, \frac{\partial f}{\partial x_{\ell}}\right\rangle
$$

the $\mathscr{O}_{U}$-ideal generated by the partial derivatives of $f$. Note that $\mathscr{J}_{U, f}$ varies with $f$ even if $U, Y$ and $\boldsymbol{x}$ are fixed. (Example: $f_{1}=x-1$ and $f_{2}=x^{2}-1$ on $\left.\mathbb{C}^{1} \backslash \operatorname{Var}(x+1)\right)$. The 1-st Fitting ideal of $\Omega_{U \cap Y}^{1}$ is

$$
\mathscr{J}_{U, Y}=\mathscr{J}_{U, f} \cdot \mathscr{O}_{U \cap Y} \text {. }
$$

In contrast to $\mathscr{J}_{U, f}, \mathscr{J}_{U, Y}$ only depends on $U$ and $Y$, and the various $J_{U, Y}$ patch to an ideal sheaf $\mathscr{J}_{Y}$ on $Y$. We call the subscheme

$$
Z=\operatorname{Var}\left(\mathscr{J}_{Y}\right) \subseteq Y
$$

with structure sheaf $\mathscr{O}_{Y} / \mathscr{J}_{Y}$ the Jacobian scheme of $Y$. We shall use $\mathscr{I}_{X, Z}$ for the preimage ideal sheaf of $\mathscr{J}_{Y}$ under the natural projection

$$
\iota^{\#}: \mathscr{O}_{X} \rightarrow \iota_{*} \mathscr{O}_{Y} .
$$

Write Syz $\mathscr{J}_{U, f}$ for the syzygy sheaf of $\mathscr{J}_{U, f}$. We freely use the obvious identification between this sheaf with the vector fields on $U$ that annihilate $f$. Geometrically, its elements are vector fields tangent to the smooth part of all level sets of $f$. Like $\mathscr{J}_{U, f}$, Syz $\mathscr{J}_{U, f}$ varies with $f$, even if $U$ and $\boldsymbol{x}$ are fixed. For an arrangement $Y=\mathscr{A}$ in $X=V$, this definition agrees with $D^{(1, \ldots, 1)}(\mathscr{A})$ from [9]: we develop this further in $\S 1.3$.

There is a commutative diagram in the category of $\mathscr{O}_{X}$-modules

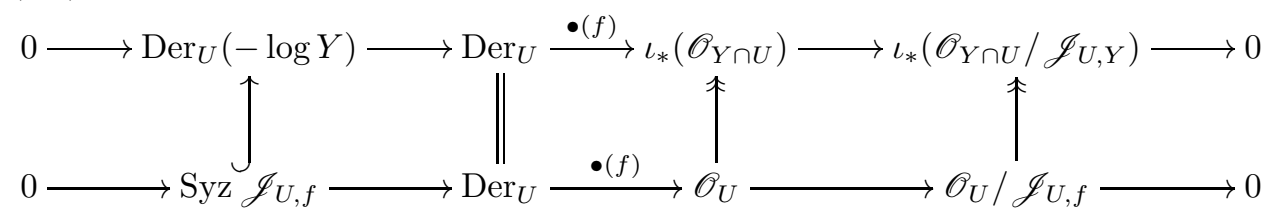

with exact rows. Here, $\bullet(f)$ denotes application to $f$.

Definition 1.4. We say that $Y$ admits an Euler vector field $\chi \in \operatorname{Der}_{U}$ on the open set $U \subseteq X$ if $\chi(f)=f$ for some local reduced defining equation $f \in \mathscr{O}_{X}(U)$ of $Y \cap U$. If $Y$ can be covered by such open sets $U$, we call $Y$ Euler homogeneous.

Let us suppose now that $Y$ admits a global Euler vector field $\chi$ on $X$. This has two important consequences. Firstly, for any local defining equation $f$ on any open affine $U, f$ is an element of $\mathscr{J}_{U, f}$. It follows that $\mathscr{J}_{U, f}=\mathscr{I}_{X, Z}$ depends only on $U$ and $Y$ but not on the choice of $f \in \mathscr{O}_{X}(U)$ or the coordinate system $\boldsymbol{x}$; in particular, one can extend diagram (1.2) to all of $X$. Secondly, the map

$$
\operatorname{Der}_{U}(-\log Y) \ni \delta \mapsto \delta-\frac{\delta(f)}{f} \cdot \chi \in \operatorname{Syz} \mathscr{J}_{U, f}
$$

defines a non-canonical local splitting of the vertical inclusion in (1.2). Thus, there is a split exact sequence of sheaves

$$
0 \longrightarrow \operatorname{Syz} \mathscr{J}_{U, f} \longrightarrow \operatorname{Der}_{U}(-\log Y) \longrightarrow \mathscr{O}_{U} \cdot \chi \longrightarrow 0 .
$$


For an arrangement $Y=\mathscr{A}$ in $X=V, \operatorname{Der}(-\log Y) / \mathscr{O} \cdot \chi=D_{0}(\mathscr{A})$ in the notation of [9].

1.3. Arrangements. We introduce the family of divisors that we are most interested in and refer to [23] for further details.

Notation 1.5. By an arrangement we mean a finite union $\mathscr{A}$ of hyperplanes in affine space. Throughout, we assume that arrangements are central, involving $n$ hyperplanes in $V$ passing through the origin. It is easy to see that $D(\mathscr{A})$ and $\Omega^{p}(\mathscr{A})$ are graded modules in this case. Abusing notation, we view $\mathscr{A}$ both as a divisor $Y=\bigcup_{H \in \mathscr{A}} H$, and as a list of hyperplanes.

For $H \in \mathscr{A}$, let $\alpha_{H} \in V^{*}$ be a defining linear form. Then

$$
f=\prod_{H \in \mathscr{A}} \alpha_{H}
$$

is a defining equation of $\mathscr{A}$ on $V$, and we call

$$
J_{\mathscr{A}}=\left\langle\frac{\partial f}{\partial x_{1}}, \ldots, \frac{\partial f}{\partial x_{\ell}}\right\rangle \subseteq S
$$

the Jacobian ideal of $\mathscr{A}$. In the case where $Y=\mathscr{A}$ is an arrangement, in order to streamline the notation and following an established convention, we write $D(\mathscr{A})$ for $\operatorname{Der}(-\log Y)$, and $\Omega^{p}(\mathscr{A})$ for $\Omega^{p}(\log Y)$.

Remark 1.6. Since $\mathscr{A}$ is a cone over the origin, we can consider its image $\mathbb{P} \mathscr{A}$ in $\mathbb{P}_{\mathrm{K}}^{\ell-1}$. By choosing a hyperplane $H \in \mathscr{A}$, we choose an affine chart in $\mathbb{P}_{\mathrm{K}}^{\ell-1}$ given by $\alpha_{H} \neq 0$. The restriction of $\mathbb{P} \mathscr{A}$ to such a chart is the noncentral arrangement of hyperplanes in $\mathbb{A}^{\ell-1}$ given by dehomogenizing $f$ relative to $H$. By reversing this construction, many questions regarding general hyperplane arrangements can be reduced to the case of central arrangements (see, e.g., [23, §3.2]).

Recall that a flat $F$ of an arrangement $\mathscr{A}$ is an intersection of hyperplanes. The intersection lattice $L(\mathscr{A})$ is the set of all flats, partially ordered by reverse inclusion.

Proposition 1.7. Let $p=$ char $\mathbb{K}$. A hyperplane arrangement $\mathscr{A}$ is an Eulerhomogeneous divisor provided that $p$ does not divide

$$
\operatorname{lcm}_{F \in L(\mathscr{A})}|\{H \in \mathscr{A}: H \leq F\}| .
$$

Proof. For a point $x \in \mathscr{A}$, let $F_{x}$ be the set-theoretically smallest flat containing $x$. Let $k$ be the number of hyperplanes containing $F_{x}$. The divisor is defined locally by a homogeneous polynomial of degree $k$, so the derivation $1 / k \sum_{i=1}^{\ell} x_{i} \partial / \partial x_{i}$ is an Euler vector field in the neighborhood of $x$, provided that $p \nmid k$.

One then naturally arrives at:

Definition 1.8. If $\mathscr{A}$ is an arrangement over a field $\mathbb{K}$, say $p=$ char $\mathbb{K}$ is $\operatorname{good}$ for $\mathscr{A}$ if $p$ does not divide the expression (1.4).

Definition 1.9. If the complement in $X$ of the free locus of $Y$ is zero-dimensional, we say that $Y$ is free outside points. If $X=\mathbb{A}_{\mathrm{KK}}^{\ell}$, and if $Y$ is quasi-homogeneous and free outside points then the origin is the only non-free point of $Y$ and we then call $Y$ free outside 0. 
Remark 1.10. If $Y$ is a central hyperplane arrangement in affine space, then the traditional term for $Y$ being free outside points is "locally free". This terminology is traditional in arrangement theory, and is rooted in Remark 1.6: for any homogeneous $Y \subset V$, the sheaf $\operatorname{Der}(-\log \mathbb{P} Y)$ on $\mathbb{P}_{\mathrm{K}}^{\ell-1}$ is locally free if and only if $Y$ is free outside the origin of $\mathbb{A}_{\mathrm{K}}^{\ell}$ in the above sense.

In Section 3, we investigate general homological properties of $\Omega^{\bullet}(\log Y)$. One natural such property, a relaxation of freeness and inspired by the arrangement case, is that of tameness:

Definition 1.11. The divisor $Y \subset X$ is called tame if $\operatorname{pdim}_{\mathscr{O}_{X, x}} \Omega^{p}(\log Y) \leq p$ for all $x \in X$ and all $0 \leq p \leq \ell$.

Many interesting families of hyperplane arrangements are tame; these include all arrangements in $\mathbb{A}^{3}$; generic arrangements; supersolvable and reflection arrangements (the last two of which are, in fact, free). The tame hypothesis appears explicitly first in [25] and frequently since. In particular, tame arrangements satisfy a Logarithmic Comparison Theorem [36]. More discussion and another application is found in [7].

We introduce in Section 2 a weaker version of tameness and, with the general results in Section 3, state a criterion that forces an Euler-homogeneous divisor $Y$ to be free provided it is free outside points and has a certain tameness property.

In Section 4 , we investigate the case of a generic central arrangement $\mathscr{A}$ and determine formulas describing the Hilbert function of the modules $\operatorname{Ext}_{S}^{i}\left(\Omega^{p}(\mathscr{A}), S\right)$ for any $p$ and $i$.

Our hope is that this article serves to trigger more studies on the interplay of homological properties of the logarithmic $p$-forms of arrangements and their combinatorics.

\section{EMBEDDED PRIMES OF THE JACOBIAN IDEAL}

Our hypotheses on $Y$, when combined with the Jacobian criterion for smoothness, imply that $\mathscr{I}_{X, Z}$ has codimension at least 2 . If the divisor $Y$ is not smooth, the upper row of display (1.2) shows that $\operatorname{Der}(-\log Y)$ is a vector bundle if and only if $\mathscr{I}_{X, Z}$ is a Cohen-Macaulay ideal of codimension two. For this to happen, $\mathscr{I}_{X, Z}$ must not have embedded primes. In this section, we study the converse of this implication for $Y$ that is free outside points under two additional hypotheses: Euler homogeneity, and a weakened form of tameness.

2.1. The algebraic case. For an Euler homogeneous divisor $Y$, we obtain from (1.2) the following identifications if $i>0$ :

$$
\mathscr{E} x t_{\mathscr{O}}^{i}(\operatorname{Der}(-\log Y), \mathscr{O}) \cong \mathscr{E} x t_{\mathscr{O}}^{i}\left(\operatorname{Syz} \mathscr{J}_{U, f}, \mathscr{O}\right) \cong \mathscr{E} x t_{\mathscr{O}}^{i+2}\left(\mathscr{O}_{Z}, \mathscr{O}\right)
$$

Lemma 2.1. The following are equivalent:

(1) $Z$ is of pure codimension 2 in $X$.

(2) $\operatorname{codim}_{X} \operatorname{supp} \mathscr{E} x t_{\mathscr{O}}^{p}\left(\mathscr{O}_{Z}, \mathscr{O}\right)>p$, for $p \geq 3$.

If $Y$ is Euler homogeneous then the above conditions are equivalent to:

(3) $\operatorname{codim}_{X} \operatorname{supp} \mathscr{E} x t_{\mathscr{O}}^{p}(\operatorname{Der}(-\log Y), \mathscr{O})>p+2$, for $p \geq 1$.

Proof. By [11, Thm. 1.1], a prime ideal of codimension $p+2$ is associated to $\mathscr{I}_{X, Z}$ if and only if it is contained in the support of $\mathscr{E} x t_{\mathscr{O}}^{p+2}\left(\mathscr{O}_{Z}, \mathscr{O}\right)=\mathscr{E} x t_{\mathscr{O}}^{p}(\operatorname{Der}(-\log Y), \mathscr{O})$. 
We can express freeness outside points homologically in terms of $\mathscr{E} x t$-sheaves as follows.

Lemma 2.2. The following are equivalent:

(1) $Y$ is free outside points.

(2) $\mathscr{E} x t_{\mathscr{O}}^{p}\left(\mathscr{E} x t_{\mathscr{O}}^{q}\left(\Omega^{1}(\log Y), \mathscr{O}\right), \mathscr{O}\right)=0$ if both $q>0$ and $p<\ell$.

Proof. The statement can be verified locally at a point $x \in X$. So we may replace $Y$ by its germ at $x$ and $\mathscr{O}$ by $R=\mathscr{O}_{x}$. If $Y$ is free outside points but not free, then all associated primes of $\operatorname{Ext}_{R}^{q}\left(\Omega^{1}(\log Y), R\right)$ are maximal ideals for $q>0$. Then by Ischebeck's Theorem (see [20, Thm. 17.1]), only $\operatorname{Ext}_{R}^{\ell}\left(\operatorname{Ext}_{R}^{q}\left(\Omega^{1}(\log Y), R\right), R\right)$ can be non-zero. Conversely, if $Y$ is not free outside points, then there is a minimal prime $\mathfrak{p}$ of codimension $p<\ell$, and a $q>0$ for which $\operatorname{Ext}_{R}^{q}\left(\Omega^{1}(\log Y), R\right)_{\mathfrak{p}} \neq 0$. By [11, Thm. 1.1], $\mathfrak{p}$ is then associated to $\operatorname{Ext}_{R}^{p}\left(\operatorname{Ext}_{R}^{q}\left(\Omega^{1}(\log Y), R\right), R\right)$ which cannot be zero in this case.

In order to relate the Ext-modules in Lemmas 2.1 and 2.2, we use a double-dual spectral sequence.

Remark 2.3. The following construction, a Grothendieck spectral sequence obtained by composing the functor $\operatorname{Hom}_{R}(-, R)$ with itself, appears already in [24]. Suppose $R$ is a regular ring. Let $0 \longrightarrow F_{\bullet} \longrightarrow M \longrightarrow 0$ be a projective resolution of $M$ with dual complex $\operatorname{Hom}_{R}\left(F_{\bullet}, R\right)=\left(0 \longrightarrow F_{0}^{\vee} \longrightarrow \cdots \longrightarrow F_{d}^{\vee} \longrightarrow 0\right)$. Let $C_{\bullet}, \bullet$ be a projective Cartan-Eilenberg resolution of $F_{\bullet}^{\vee}$. Consider the two usual spectral sequences to the dual double complex $E_{\bullet}^{0} \bullet=\operatorname{Hom}_{R}(C \bullet \bullet, \bullet, R)$. The total complex of $E$ is a representative of $\mathbb{R} \operatorname{Hom}_{R}\left(\mathbb{R} \operatorname{Hom}_{R}(M, R), R\right) \cong M$. Thus, the spectral sequences converge to $M$.

The second spectral sequence has, with suitable choice of indices, the differentials $d^{0}$ oriented downward and $d^{1}$ oriented to the left, and

$$
E_{p, q}^{2}=\operatorname{Ext}_{R}^{-p}\left(\operatorname{Ext}_{R}^{q}(M, R), R\right) \Longrightarrow M .
$$

With this choice, the abutment $M$ is regarded as a chain complex concentrated in total degree 0 .

Proposition 2.4. If $Y$ is free outside points, then there are isomorphisms

$$
\mathscr{E} x t_{\mathscr{O}}^{\ell-p-1}(\operatorname{Der}(-\log Y), \mathscr{O}) \longrightarrow \mathscr{E} x t_{\mathscr{O}}^{\ell}\left(\mathscr{E} x t_{\mathscr{O}}^{p}\left(\Omega^{1}(\log Y), \mathscr{O}\right), \mathscr{O}\right), \quad p>0 .
$$

Proof. As we will see below, over any affine open subset $U \subset X$, these isomorphisms are morphisms in the Grothendieck spectral sequence in Remark 2.3 for $R=\mathscr{O}(U)$. By functoriality of this spectral sequence, these patch together to an isomorphism defined on $X$.

The spectral sequence (2.2), applied to $M=\Omega^{1}(\log Y)(U)$, is illustrated in Figure 1. By Lemma 2.2, the only non-zero entries are in column $p=-\ell$, or in row $q=0$. Let $R=\mathscr{O}(U)$, and omit the argument " $U$ " in all instances of global sections below. For $q=0$, we have $E_{-p, 0}^{2}=\operatorname{Ext}_{R}^{p}(\operatorname{Der}(-\log Y), R)$, which is non-zero only if $p \leq \ell-2$ since depth $\operatorname{Der}(-\log Y) \geq 2$ by reflexivity of $\operatorname{Der}(-\log Y)$. As $\Omega^{1}(\log Y)$ is reflexive, $E_{0,0}^{2}=\Omega^{1}(\log Y)$. Hence, the only possible non-zero higher differentials $d^{p+1}: E_{-\ell+p+1,0}^{p+1} \longrightarrow E_{-\ell, p}^{p+1}$ must be isomorphisms

$$
d^{r}: \operatorname{Ext}_{R}^{\ell-p-1}(\operatorname{Der}(-\log Y), R) \longrightarrow \operatorname{Ext}_{R}^{\ell}\left(\operatorname{Ext}_{R}^{p}\left(\Omega^{1}(\log Y), R\right), R\right) .
$$


FiguRe 1. $E_{p q}^{2}$

\begin{tabular}{|cccccc|c|}
\hline$*$ & 0 & $\ldots$ & 0 & 0 & 0 & $\ell-2$ \\
$\vdots$ & $\vdots$ & & $\vdots$ & $\vdots$ & $\vdots$ & $\vdots$ \\
$*$ & 0 & 0 & $\ldots$ & 0 & 0 & 1 \\
0 & 0 & $*$ & $\cdots$ & $*$ & $\Omega^{1}(\log Y)$ & 0 \\
\hline$-\ell$ & $-(\ell-1)$ & $-(\ell-2)$ & $\ldots$ & -1 & 0 & $\mathrm{p}$ \\
\hline
\end{tabular}

Adding a weakened form of tameness as a second additional hypothesis, we obtain the following result:

Theorem 2.5. Suppose that $Y$ is free outside points and Euler homogeneous, and that $\operatorname{pdim}_{\mathscr{O}_{x}} \Omega^{1}(\log Y)_{x} \leq 1$ for all $x \in Y$. Then $Y$ is free if and only if $Z$ is pure of codimension 2 in $X$.

Proof. All plane curves are free, so assume $\ell \geq 3$.

The statement is clearly local, so we may replace $Z \subseteq Y \subseteq X$ by their germs at $x \in Z$ and work over $R=\mathscr{O}_{x}$. As $\operatorname{pdim}_{R} \Omega^{1}(\log Y) \leq 1$, we have $E_{p, q}^{2}=0$ for $q \geq 2$ in (2.2) for $M=\Omega^{1}(\log Y)_{x}$. By Proposition 2.4, $\operatorname{Ext}_{R}^{p}(\operatorname{Der}(-\log Y), R)=0$ unless $p=0, \ell-2$.

Since $Z$ is pure of codimension 2 in $X$, Lemma 2.1 implies

$$
\operatorname{codim}_{X} \operatorname{supp} \operatorname{Ext}_{R}^{\ell}(\operatorname{Der}(-\log Y), R)>\ell=\operatorname{dim} R .
$$

Therefore, $\operatorname{Ext}_{R}^{\ell}(\operatorname{Der}(-\log Y), R)=0$ for all $p>0$ and hence $\operatorname{Der}(-\log Y)$ is free as claimed.

2.2. Arrangements. By Proposition 1.7, hyperplane arrangements in good characteristic provide a family of examples of Euler homogeneous divisors. In this case we can allow a larger non-free locus.

Notation 2.6. If $F$ is a flat of an arrangement, then $\mathscr{A}_{F}$ is the arrangement of all hyperplanes containing $F$.

Corollary 2.7. Let $\mathscr{A}$ be a hyperplane arrangement in good characteristic with $\operatorname{pdim}_{S} \Omega^{1}(\mathscr{A}) \leq 1$. If $J_{\mathscr{A}}$ is pure of codimension 2 then $\mathscr{A}$ is free.

Proof. As the statement is local, we may assume that $\mathscr{A}$ is central. Let $X_{0} \in L(\mathscr{A})$ be a flat such that $\Omega^{1}\left(\mathscr{A}_{X^{\prime}}\right)$ is free for all flats $X^{\prime} \supsetneq X_{0}$; then it suffices to prove that $\Omega^{1}\left(\mathscr{A}_{X_{0}}\right)$ is free.

Both $\mathscr{A} \mapsto \Omega^{1}(\mathscr{A})$ and $\mathscr{A} \mapsto J_{\mathscr{A}}$ are local functors in the sense of [31], so we may replace $\mathscr{A}$ by $\mathscr{A}_{X_{0}}$.

Now $\mathscr{A}$ is the product of its essentialization $\mathscr{A}_{0}$ (in $X / X_{0}$ ) and the affine space $X_{0}$. Then $\Omega^{1}(\mathscr{A})$ is the direct sum of the free $S$-modules $\mathscr{O}_{X / X_{0}}\left(X / X_{0}\right) \otimes_{\mathbb{K}} \Omega_{X_{0}}^{1}$ and $\mathscr{O}_{X_{0}}\left(X_{0}\right) \otimes_{\mathbb{K}} \Omega_{X / X_{0}}^{1}\left(\mathscr{A}_{0}\right)$, these being $S$-modules via $S \rightarrow \mathscr{O}_{X_{0}}\left(X_{0}\right)$ and $S \rightarrow$ $\mathscr{O}_{X / X_{0}}\left(X / X_{0}\right)$. Moreover, $J_{\mathscr{A}}$ and $J_{\mathscr{A}_{0}}$ have the same generators in a generic point of $X$. We may therefore replace $\mathscr{A}$ by $\mathscr{A}_{0}$. 
Now $\mathscr{A}$ is free outside points and hence free by Theorem 2.5 .

Remark 2.8.

(1) The hypothesis that $\operatorname{pdim}_{S} \Omega^{1} \leq 1$ is necessary. The Edelman-Reiner example [10] defined by

$$
Q=\prod_{\alpha \in\{0,1\}^{4}, \alpha \neq(0,0,0,0)}\left(\alpha_{1} x_{1}+\alpha_{2} x_{2}+\alpha_{3} x_{3}+\alpha_{4} x_{4}\right)
$$

is free outside the origin, its Jacobian ideal has no embedded primes, yet it is not free. Here, $\operatorname{pdim}_{S} \Omega^{1}(\mathscr{A})=2$.

(2) Add a generic hyperplane $x_{5}=0$ to the example $\mathscr{A}$ above to obtain an arrangement $\mathscr{B}$ defined by $Q x_{5}$. The associated primes of $J\left(Q x_{5}\right)=\left(Q, x_{5} J(Q)\right)=$ $\left(Q, x_{5}\right) \cap J(Q)$ are all codimension 2 , since $J(Q)$ has no submaximal embedded components. Nevertheless, $\mathscr{B}$ is not free outside points, since $\mathscr{A}$ defines a nonmaximal, non-free flat of $\mathscr{B}$. Again, $\operatorname{pdim} \Omega^{1}(\mathscr{B})=2$.

(3) One way of reading (the proof of) Corollary 2.7 is: "if $\operatorname{pdim}_{S} \Omega^{1}(\mathscr{A}) \leq 1$ then the top-dimensional non-free flats of $\mathscr{A}$ are the minimal embedded primes of $J_{\mathscr{A}}$."

(4) Let $\mathscr{A}$ be of rank three. Then $\mathscr{A}$ is free outside points and $\operatorname{pdim}_{S} \Omega^{1} \leq 1$ due to reflexivity. Thus, in this case Theorem 2.5 becomes the well-known statement "Ad is free if and only if $J_{\mathscr{A}}$ has no embedded primes."

(5) The hypothesis that $J_{\mathscr{A}}$ has no embedded primes is necessary. Generic arrangements of $n>\ell$ hyperplanes are free outside points. If $\ell \geq 3$, they have $\operatorname{pdim}_{S} \Omega^{1}(\mathscr{A})=1$ by [39, Cor. 7.7], so they are not free.

Remark 2.9. In [22], Proposition 2.10 and 2.7, it is shown that if $\operatorname{dim} X \geq p-1$ then freeness of $D_{\mathscr{A}_{X}}^{p}$ is equivalent to freeness of $\mathscr{A}_{X}$.

2.3. Analytic divisors. We aim for a generalization of Corollary 2.7 to more general divisors. For the desired analogue of the stratification by flats of an arrangement we need to move to the analytic category. So let $X$ be a complex $\ell$-dimensional analytic manifold.

The stratification by flats for arrangements is a special case of the logarithmic stratification $\left\{Y_{\alpha}\right\}$ along $Y$ introduced by K. Saito [27, §3]. It is uniquely characterized by the following properties [27, Lemma 3.2]:

(1) Each stratum $Y_{\alpha}$ is a smooth connected immersed submanifold of $X$, and $X$ is their disjoint union.

(2) If $x \in Y_{\alpha}$ then the tangent space $T_{Y_{\alpha}, x}$ coincides with the space $\operatorname{Der}(-\log Y)_{x}$ of logarithmic vector fields evaluated at $x$.

(3) If $Y_{\alpha}$ meets the closure of $Y_{\beta}$ in $X$ with $\alpha \neq \beta$ then $Y_{\alpha}$ is in the boundary of $Y_{\beta}$.

Note that $Y, X \backslash Y$, and $\operatorname{Sing}(Y)$ are (unions of) logarithmic strata. The term "logarithmic stratification" is a misnomer as it is not locally finite in general.

Example 2.10 ([4, Rem. 4.2.4]). Each point on the $z$-axis is a logarithmic stratum of the free divisor $Y=(x y(x+y)(x+z y))$.

Definition 2.11 ([27, Def. 3.8]). The divisor $Y$ is called holonomic (at $x \in X$ ), if its logarithmic stratification is locally finite (at $x$ ). For free $Y$, holonomicity is also referred to as Koszul freeness. 
By [27, (3.10.i),(3.13.(ii)], if $Y$ is holonomic at $x \in Y_{\alpha}$ then the manifold topology of $Y_{\alpha}$ is the induced topology from $X$ near $x$; this can fail in general.

Saito introduced the logarithmic characteristic variety $L_{Y}(\log Y) \subset T_{X}^{*}$, defined by the symbols of $\operatorname{Der}(-\log Y)$ with respect to the order filtration $F_{\bullet}$ on $\mathscr{D}_{X}$. He showed that holonomicity is (locally) equivalent to $L_{Y}(-\log Y)$ having minimal dimension $\ell$ [27, (3.18)]. In particular, Saito's holonomicity implies that $M^{\log Y}=$ $\mathscr{D}_{X} / \mathscr{D}_{X} \cdot \operatorname{Der}(-\log Y)$ is a holonomic $D$-module in the sense of Kashiwara. This implication cannot be reversed, as we were informed by F. Castro Jiménez:

Example 2.12. The divisor $Y$ given by $(x z+y)\left(x^{4}+y^{5}+x y^{4}\right)$ is not holonomic in the sense of Saito, but $M^{\log Y}$ is a holonomic $D$-module.

By $[27,(3.6)]$, the logarithmic stratification provides us with the local product structures that we used in the proof of Corollary 2.7:

Proposition 2.13. Let $x \in Y_{\alpha}$ and set $m=\operatorname{dim} Y_{\alpha}$. On small neighborhoods $U$ of $x, Y \cap U \subseteq U$ is defined by some $f$ such that $f(z)=f\left(z_{1}, \ldots, z_{\ell-m}, 0, \ldots, 0\right)$ and $Y_{\alpha} \cap U_{x}=\left\{z_{1}=\cdots=z_{\ell-m}=0\right\}$ for some coordinate system $z_{1}, \ldots, z_{\ell}$ on $U$. In other words, there is an isomorphism of pairs of germs

$$
\left(Y_{x}, X_{x}\right) \cong\left(Y_{0}^{\prime}, \mathbb{C}_{0}^{\ell-m}\right) \times\left(Y_{\alpha, x}, Y_{\alpha, x}\right), \quad Y_{\alpha, x} \cong \mathbb{C}_{0}^{m}
$$

This isomorphism identifies the logarithmic stratifications of $Y_{x}$ and $Y_{0}^{\prime}$.

By connectedness of the strata this implies in particular:

Corollary 2.14. Freeness of $Y$ at $x \in Y_{\alpha}$ is a property of $Y_{\alpha}$.

There is a similar corollary for holonomicity, and the notion of a holonomic stratum, but we do not need it (see [27, (3.10) Prop. i)]).

Definition 2.15. We call $Y_{\alpha}$ a free stratum if $Y$ is free at some $x \in Y_{\alpha}$.

Euler homogeneity does not descend in general to factors in a product; in fact, products with analytic affine spaces are always Euler homogeneous.

Example 2.16. Let $Y=Y^{\prime} \times \mathbb{A}_{\mathbb{C}}^{1}$ with $Y^{\prime}=(f(x)) \subseteq \mathbb{A}_{\mathbb{C}}^{\ell-1}$. Then $e^{y} \cdot f(x)$ is a defining equation for $Y$ and $\chi=\frac{\partial}{\partial y}$ is an Euler vector field for $Y$. So $Y$ is Euler homogeneous. However, choosing $Y^{\prime}$ with an isolated non-quasihomogeneous singularity, it is not Euler homogeneous by Saito's theorem [26].

We need the following stronger version of Euler homogeneity which is well-known in the context of the Logarithmic Comparison Theorem for free divisors (see [5, Conj. 1.4] and, for example, [14, 13, 15]).

Definition 2.17. The divisor $Y$ is called strongly Euler homogeneous if it admits, at each point $x \in X$, an Euler vector field vanishing at $x$.

By [14, Lem. 3.2], strong Euler homogeneity for $Y$ and $Y^{\prime}$ in (2.4) are equivalent.

Theorem 2.18. Let $Y$ be a strongly Euler homogeneous and holonomic divisor in a complex manifold $X$ such that pdim $\mathscr{O}_{x} \Omega^{1}(\log Y)_{x} \leq 1$ for all $x \in X$. Then $Y$ is free if $Z$ is of pure of codimension 2 in $X$.

Proof. We proceed along the lines of the proof of Corollary 2.7.

Let $Y_{\alpha}$ be a stratum such that all strata $Y_{\beta}$ containing $Y_{\alpha}$ in their closure are free. To show that $Y_{\alpha}$ is free, we may replace $Z \subset Y \subset X$ by their germs at $x \in Z$ and work over $R=\mathscr{O}_{x}$. 
Using Proposition 2.13, we may replace $Y$ by $Y^{\prime}$ in (2.4), and hence assume that $Y$ is free outside points. Here we use that strong Euler homogeneity is preserved by [14, Lem. 3.2], freeness and $\operatorname{pdim}_{R} \Omega^{1}(\log Y)$ are preserved by [6, Lem. 2.2.(iv)], and $Z=Z^{\prime} \times Y_{\alpha, x}$ where $Z^{\prime}$ is the Jacobian scheme of $Y^{\prime}$ in (2.4).

Finally, we apply Theorem 2.5 whose proof works also in the analytic setup. Thus, $Y_{\alpha}$ is free and the claim then follows by descending induction on $\operatorname{dim} Y_{\alpha}$.

\section{HomologicAl PROPERTIES OF LOGARITHMiC FORMS}

The previous section indicates the importance of the condition pdim $\operatorname{dim}_{\mathscr{O}} \Omega^{1}(\log Y) \leq$ 1. The results in the present section come from an attempt to understand what can be rescued if this hypothesis on $\operatorname{pdim}_{\mathscr{O}} \Omega^{1}(\log Y)$ is false or unknown. Specifically, we are interested in the difference between $\Omega^{p}(\log Y)$ and $\bigwedge^{p} \Omega^{1}(\log Y)$. Recall that $M^{\vee}=\operatorname{Hom}_{R}(M, R)$ for any module.

Convention 3.1. Throughout this section we assume that, if $\mathscr{A}$ is an arrangement of rank $\ell$ over a field $\mathbb{K}$, then char $\mathbb{K}$ is either zero, or both good (Definition 1.8) and at least $\ell$.

3.1. Higher forms as exterior products. The exterior product gives an exact sequence

$$
\bigwedge^{p} \Omega^{1}(\log Y) \stackrel{j_{p}}{\longrightarrow} \Omega^{p}(\log Y) \longrightarrow E_{Y}^{p}:=\frac{\Omega^{p}(\log Y)}{\bigwedge^{p} \Omega^{1}(\log Y)} \longrightarrow 0
$$

Saito [27] showed that in all free points of $Y, j_{p}$ is an isomorphism for all $p$; see also [9, formula (2.3)]. In general, one knows from [9, Prop. 2.2] that $\Omega^{p}(\log Y)$ is the reflexive hull $\left(\bigwedge^{p} \Omega^{1}(\log Y)\right)^{\vee \vee}$ of $\bigwedge^{p} \Omega^{1}(\log Y)$.

Given some information about the codimension of the non-free locus, it is possible to say more. Mustaţa and Schenck prove the following for arrangements in [22]:

Theorem 3.2. Let $\mathscr{A}$ be free outside points and $\operatorname{pdim}_{S} \Omega^{1}(\mathscr{A})=1$. Then, for $0 \leq p \leq \ell-2, j_{p}$ in (3.1) is an isomorphism and $\operatorname{pdim}_{S} \Omega^{p}(\mathscr{A})=p$. Moreover, $\operatorname{pdim}_{S} \Omega^{\ell-1}(\mathscr{A})=\ell-2$.

Example 3.3. The rank-4 arrangement $\mathscr{A}$ of [7, Example 5.3] is free outside points and $\operatorname{pdim}_{S} \Omega^{1}(\mathscr{A})=2$. Further calculation shows that the map $\bigwedge^{2} \Omega^{1}(\mathscr{A}) \longrightarrow$ $\Omega^{2}(\mathscr{A})$ is injective, but not an isomorphism. Moreover, the map $j_{p}$ need not be injective in general: here, for example, $\bigwedge^{5} \Omega^{1}(\mathscr{A})$ is a (nonzero) torsion module supported at the maximal ideal, while $\Omega^{5}(\log \mathscr{A})=0$.

Definition 3.4. For $k \in \mathbb{N}$, we call the divisor $Y k$-tame if pdim $\mathscr{O}_{X, x} \Omega^{p}(\log Y)_{x} \leq$ $p$ for all $x \in X$ and all $0 \leq p \leq k$.

In particular, by Theorem 3.2, an arrangement free outside points is tame if and only if it is 1-tame. Denham and Schulze in [9, Prop. 2.9] give the following variation:

Theorem 3.5. If the codimension of the non-free locus of $\mathscr{A}$ is greater than $k$ and $\mathscr{A}$ is $(k-1)$-tame, then the map $j_{p}$ of (3.1) is an isomorphism for $0 \leq p<k$.

As a matter of fact, inspection of the proof of [9, Prop. 2.9] reveals that $\mathscr{A}$ need not be an arrangement but can be an arbitrary $(k-1)$-tame divisor with non-free locus of codimension $k+1$ or more. 
Problem 3.6. Describe in general the modules $E_{Y}^{p}$, or at least their vanishing.

We now prepare the way for a different strengthening (Corollary 3.13 below) of Theorem 3.2, this time relaxing the 1-tameness condition while adhering to the case of divisors free outside points. We first prove general statements on reflexive modules with zero-dimensional non-free locus; these involve the following technical definitions. The first definition can be found for instance in [16].

Definition 3.7. An $R$-module $M$ is an $r$-syzygy (of $N$ ) if there is an exact sequence

$$
0 \longrightarrow M \longrightarrow P_{r} \longrightarrow \ldots \longrightarrow P_{1}(\longrightarrow N \longrightarrow 0)
$$

where each $P_{i}$ is $R$-projective. On the other hand, $M$ is $k$-torsion free if every $R$-regular sequence of length $\leq k$ is also $M$-regular.

Being an $r$-syzygy implies $r$-torsion freeness. The two notions are equivalent, if $\operatorname{pdim}_{R} M<\infty$ (see [17, p.2]). The next definition is due to Auslander [19]:

Definition 3.8. An $R$-module $M$ is $p$-spherical if $\operatorname{pdim}_{R} M \leq p$ and $\operatorname{Ext}_{R}^{i}(M, R)=$ 0 for $1 \leq i \leq p-1$.

For example, $R / I$ is $p$-spherical if and only if $I$ is Cohen-Macaulay of codimension $p$.

Proposition 3.9. Let $R$ be an $\ell$-dimensional regular ring. Let $M$ be a finitely generated reflexive non-free $R$-module with c-dimensional non-free locus, of projective dimension $d=\operatorname{pdim}_{R} M$. Then

$$
\operatorname{pdim}_{R} M+\operatorname{pdim}_{R} M^{\vee} \geq \ell-1-c .
$$

In case of equality, $M$ and $M^{\vee}$ are $d$ - and $(\ell-d-1-c)$-spherical, respectively.

Proof. We apply the spectral sequence of Remark 2.3, using our additional hypotheses. Since $\operatorname{Ext}_{R}^{q}(M, R)$ is Noetherian and supported for $q>0$ only in dimension at most $c$, then $E_{p, q}^{2}=0$ unless $q=0$ or $p \leq c-\ell$. Since $M$ is reflexive, $E_{0,0}^{2}$ is the abutment $M$. So $E_{\infty}^{p, q}=0$ unless $p=q=0$. Of course, $E_{2}^{p, q}=0$ for $q>d$. Now suppose $\operatorname{pdim}\left(M^{\vee}\right)=a>0$, so $E_{2}^{-a, 0} \neq 0$. Then all differentials $d_{k}^{-a, 0}$ out of $E_{k}^{-a, 0}$ end in $E_{k}^{-a-k, k-1}$. These target modules are only nonzero if $k-1 \leq d$ and simultaneously $-a-k \leq c-\ell$. Summing, the targets are zero unless (at minimum) $-a-1 \leq c-\ell+d$, i.e. $\ell-c-d-1 \leq a$. It follows that $a<\ell-c-d-1$ is impossible since it would imply $E_{\infty}^{-a, 0} \neq 0$ which we know to be false.

Suppose now that $a+d=\ell-c-1$. Then the argument above shows that $0=E_{\infty}^{-i, 0}=E_{2}^{-i, 0}$ for all $a>i>0$. Hence $M^{\vee}$ is spherical. By symmetry, the same holds for $M$. Indeed, one obtains in this case a duality:

$$
\operatorname{Ext}_{R}^{a}\left(M^{\vee}, R\right)=E_{a, 0}^{2} \cong E_{c-\ell, d}^{2}=\operatorname{Ext}_{R}^{\ell-c}\left(\operatorname{Ext}_{R}^{d}(M, R), R\right) .
$$

A special case of the above proposition is worth singling out. Suppose that $M$ is a reflexive $R$-module of projective dimension $d$, with a zero-dimensional non-free locus. Then

$$
\operatorname{pdim}_{R} M+\operatorname{pdim}_{R} M^{\vee} \geq \ell-1,
$$

and in the case of equality, $M$ and $M^{\vee}$ are $d$ - and $(\ell-d-1)$-spherical, respectively. A partial converse is the following. 
Proposition 3.10. Let $R$ be a regular ring of dimension $\ell$. Let $M$ be a d-spherical $R$-module with zero-dimensional non-free locus, where $0<d<\ell$. Then $M$ is reflexive and $M^{\vee}$ is $(\ell-d-1)$-spherical with zero-dimensional non-free locus. Moreover, $M$ and $M^{\vee}$ are $(\ell-d)$ - and $(d+1)$-syzygies, respectively.

Proof. By hypothesis, $M$ has a projective resolution of length $d>0$,

$$
0 \longleftarrow M \longleftarrow F_{0} \longleftarrow F_{1} \longleftarrow \cdots \longleftarrow F_{d} \longleftarrow 0 .
$$

Since $\operatorname{Ext}_{R}^{i}(M, R)=0$ for $0<i<d$ by hypothesis, dualizing gives an exact complex

$$
0 \longrightarrow M^{\vee} \longrightarrow F_{0}^{\vee} \longrightarrow \cdots \longrightarrow F_{d}^{\vee} \longrightarrow \operatorname{Ext}_{R}^{d}(M, R) \longrightarrow 0 .
$$

In particular, $M^{\vee}$ is a $(d+1)$-syzygy. By regularity of $R, \operatorname{pdim}_{R} \operatorname{Ext}_{R}^{d}(M, R) \leq \ell$, so this complex extends to a projective resolution

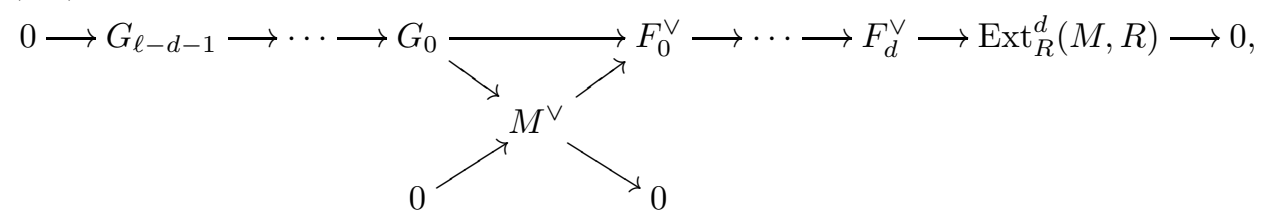

and hence $\operatorname{pdim}_{R} M^{\vee} \leq \ell-d-1$. Since $M$ has zero-dimensional non-free locus, $\operatorname{Ext}_{R}^{d}(M, R)$ is supported only at maximal ideals, and hence

$$
\operatorname{Ext}_{R}^{i}\left(\operatorname{Ext}_{R}^{d}(M, R), R\right)=0, \quad \text { for } i \neq \ell .
$$

Thus, dualizing (3.5) and comparing with (3.4) proves reflexivity of $M$. By décalage, (3.5) also shows that $\operatorname{Ext}_{R}^{i}\left(M^{\vee}, R\right)=0$ except for $i=0$ and $i=\ell-d-1$, so $M^{\vee}$ is $(\ell-d-1)$-spherical. By localization, it clearly has a zero-dimensional non-free locus. Then $M^{\vee}$ satisfies the original hypotheses and so $M$ is an $(\ell-d)$-syzygy.

For convenience, we now state a result of Lebelt [16, Satz 1].

Theorem 3.11. Let $1 \leq p \in \mathbb{N}$ and assume that the (not necessarily regular) ring $R$ contains a field $\mathbb{K}$ with $p<$ char $\mathbb{K}$ or char $\mathbb{K}=0$. If $M$ is an $R$-module with $\operatorname{pdim}_{R} M=d<\infty$ that is $(d(p-1)+k)$-torsion free for some $k \geq 0$, then $\bigwedge^{p} M$ is $k$-torsion free and $\operatorname{pdim}_{R} \wedge^{p} M \leq p d$.

In the graded case, this last inequality becomes an equality.

Theorem 3.12. Suppose $R$ is a regular ring. Let $M$ be a $d$-spherical $R$-module with zero-dimensional non-free locus. If $\ell=\operatorname{dim} R \leq \operatorname{char} \mathbb{K}$ or char $\mathbb{K}=0$ then $\bigwedge^{p} M$ is reflexive for all $p$ such that $p d<\ell-1$.

Proof. We assume $p d<\ell-1$, which is equivalent to $d(p-1)+2 \leq \ell-d$. Since $M$ is $d$-spherical, $d=\operatorname{pdim}_{R} M$. By Proposition 3.10, $M$ is $(\ell-d)$-torsion free. By Theorem 3.11 above, $\bigwedge^{p} M$ is 2-torsion free and

$$
\operatorname{pdim}_{R} \bigwedge^{p} M \leq p d<\ell-1 .
$$

Since $\bigwedge^{p} M$ is a 2-syzygy, it is a submodule of a free module and thus contained in its reflexive hull $M^{p}=\left(\left(\bigwedge^{p} M\right)^{\vee}\right)^{\vee}$. Of course, $\operatorname{pdim}_{R} M^{p} \leq \ell-2$.

Then we have a short exact sequence

$$
0 \longrightarrow \bigwedge^{p} M \longrightarrow M^{p} \longrightarrow E^{p} \longrightarrow 0 ;
$$


since $d=\operatorname{pdim}_{R} M^{p} \leq \ell-2$ as well, applying $\operatorname{Ext}_{R}^{\bullet}(-, R)$ shows that $\operatorname{Ext}_{R}^{\ell}\left(E^{p}, R\right)=$ 0 . On the other hand, $M$ has zero-dimensional non-free locus, hence $E^{p}$ is finite length, which means $\operatorname{Ext}_{R}^{q}\left(E^{p}, R\right)=0$ for $q<\ell$. It follows that $E^{p}=0$, hence $\bigwedge^{p} M \cong M^{p}$ by (3.7), which is reflexive by definition.

Corollary 3.13. Let $Y \subseteq X$ be a divisor in an $\ell$-dimensional complex manifold or smooth algebraic variety, or let $Y=\mathscr{A}$ be a hyperplane arrangement in $X=\mathbb{A}_{\mathbb{K}}^{\ell}$ with $\ell \leq$ char $\mathbb{K}$ or char $\mathbb{K}=0$. Assume that $Y$ is free outside points and that $\Omega^{1}(\log Y)_{x}$ is d-spherical for all $x \in X$. Then $\Omega^{p}(\log Y)=\bigwedge^{p} \Omega^{1}(\log Y)$ for $p d<$ $\ell-1$.

Remark 3.14. Suppose $Y$ is a homogeneous hypersurface in affine space. If $Y$ is not a free divisor, a natural question is whether any of the modules $\Omega^{i}(\log Y)$ can be free. In dimension three or less, this is impossible because of duality. In dimensions 4 and 5 , the question boils down to asking whether $\Omega^{2}(\log Y)$ can be free while $\Omega^{1}(\log Y)$ is not. One sees this to be impossible as follows: $\Omega^{2}(\log Y)$ decomposes into $\Omega_{0}^{1}(\log Y) \oplus \Omega_{0}^{2}(\log Y)$ so that freeness of $\Omega^{2}(\log Y)$ implies freeness of $\Omega_{0}^{1}(\log Y)$ and hence of $\Omega^{1}(\log Y)$. Thus, the first open case appears for $p=3$ and $\ell=6$.

\section{Generic ARrangements}

We consider here generic arrangements $\mathscr{A}$. Again, we assume that the characteristic of the base field is good (Definition 1.8). The purpose of this section is to prove Theorem 4.1 below.

Recall from [9, Def. 2.4] that the module of relative differential p-forms along $\mathscr{A}$ is the kernel $\Omega_{0}^{p}(\mathscr{A})$ of contraction with the Euler vector field $\chi$,

$$
\chi: \Omega^{p}(\mathscr{A}) \longrightarrow \Omega^{p-1}(\mathscr{A}) .
$$

These modules are non-zero only for $0 \leq p \leq \ell-1$; for $p=0$ and $p=\ell-1$ they are free of rank 1. By [9, (2.4)], we can identify

$$
\Omega^{p}(\mathscr{A}) \cong \Omega_{0}^{p-1}(\mathscr{A}) \oplus \Omega_{0}^{p}(\mathscr{A}) .
$$

So both tameness and being free outside points descends from $\Omega^{1}(\mathscr{A})$ to $\Omega_{0}^{\bullet}(\mathscr{A})$. In particular, $\Omega_{0}^{p}(\mathscr{A})$ has zero-dimensional non-free locus for $0<p<\ell-1$ if $\mathscr{A}$ is generic. Recall also the module $D_{p}^{0}(\mathscr{A})=D_{p}(\mathscr{A}) / \chi \wedge D_{p-1}(\mathscr{A})$ of relative logarithmic p-derivations along $\mathscr{A}$ defined in [9, Def. 3.4], which has the analogous property

$$
D_{p}(\mathscr{A}) \cong D_{p-1}^{0}(\mathscr{A}) \oplus D_{p}^{0}(\mathscr{A})
$$

By $\left[9\right.$, Prop. 3.5], $D_{p}^{0}(\mathscr{A})$ and $\Omega_{0}^{p}(\mathscr{A})$ are mutually $S$-dual.

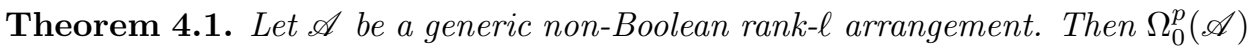
is $p$-spherical, for $0<p<\ell-1$, and $\operatorname{Ext}_{S}^{p}\left(\Omega_{0}^{p}(\mathscr{A}), S\right)$ is Artinian of length $\left(\begin{array}{c}n-1 \\ \ell\end{array}\right)$ where $n=|\mathscr{A}|$.

The proof is by induction on $n$. We first establish some lemmas in order to proceed by means of a deletion-restriction argument.

Definition 4.2. For an arrangement $\mathscr{A}$ and a hyperplane $H \in \mathscr{A}$ we denote by $\mathscr{A}^{\prime}$ the arrangement $\mathscr{A} \backslash\{H\}$ and by $\mathscr{A}^{\prime \prime}$ the arrangement induced by $\mathscr{A}^{\prime}$ on $H$. 
Now fix $H \in \mathscr{A}$ with defining equation $\alpha_{H}$. From [23, Prop. 4.45] and Ziegler [39, Cor. 4.5], there are sequences of $S$-modules

$$
\begin{gathered}
0 \longrightarrow D\left(\mathscr{A}^{\prime}\right)(-1) \stackrel{\alpha_{H}}{\longrightarrow} D(\mathscr{A}) \longrightarrow D\left(\mathscr{A}^{\prime \prime}\right)--\rightarrow 0, \\
0 \longrightarrow \Omega^{p}(\mathscr{A})(-1) \stackrel{\alpha_{H}}{\longrightarrow} \Omega^{p}\left(\mathscr{A}^{\prime}\right) \longrightarrow \Omega^{p}\left(\mathscr{A}^{\prime \prime}\right)--\rightarrow 0,
\end{gathered}
$$

which are exact except possibly at $D\left(\mathscr{A}^{\prime \prime}\right)$ and $\Omega^{p}\left(\mathscr{A}^{\prime}\right)$. Although Ziegler formulates this result only for $p=1$, it holds true in general.

Lemma 4.3. If $\mathscr{A}$ is non-Boolean generic then there are short exact sequences

$$
\begin{aligned}
& 0 \longrightarrow D^{0}\left(\mathscr{A}^{\prime}\right)(-1) \stackrel{\alpha_{H}}{\longrightarrow} D^{0}(\mathscr{A}) \longrightarrow D^{0}\left(\mathscr{A}^{\prime \prime}\right) \longrightarrow 0 \\
& 0 \longrightarrow \Omega_{0}^{\ell-2}\left(\mathscr{A}^{\prime}\right) \stackrel{\alpha_{H}}{\longrightarrow} \Omega_{0}^{\ell-2}(\mathscr{A}) \longrightarrow \Omega_{0}^{\ell-3}\left(\mathscr{A}^{\prime \prime}\right) \longrightarrow 0
\end{aligned}
$$

Proof. By Wiens [35, Thm. 3.4], (4.1) is exact at $D\left(\mathscr{A}^{\prime \prime}\right)$. By dividing out $S \chi$, this proves exactness of the first sequence. Then the second sequence is obtained via the identification $D^{0}(\mathscr{A})=\Omega_{0}^{\ell-2}(\mathscr{A})(\ell-n)$ from [9, Prop. 3.7.(3)].

Lemma 4.4. Suppose $\alpha$ is a non-zerodivisor in a ring $R$. If $M$ is a module over $R^{\prime \prime}=R / R \alpha$, then

$$
\operatorname{Ext}_{R^{\prime \prime}}^{q}\left(M, R^{\prime \prime}\right) \cong \operatorname{Ext}_{R}^{q+1}(M, R)
$$

for $q \geq 0$, and $\operatorname{Ext}_{R}^{0}(M, R)=0$. If $R$ is graded and $\alpha$ homogeneous, the isomorphism becomes graded after twisting the left hand side by $\operatorname{deg}(\alpha)$.

Proof. As an $R$-module, $R^{\prime \prime}$ has a free resolution

$$
0 \longrightarrow R \stackrel{\alpha}{\longrightarrow} R \longrightarrow R^{\prime \prime} \longrightarrow 0 .
$$

So $\operatorname{Ext}_{R}^{q}\left(R^{\prime \prime}, R\right)=0$ unless $q=1$, in which case we get $\operatorname{Ext}_{R}^{1}\left(R^{\prime \prime}, R\right)=R^{\prime \prime}$. The change-of-rings spectral sequence

$$
E_{2}^{p, q}=\operatorname{Ext}_{R^{\prime \prime}}^{p}\left(M, \operatorname{Ext}_{R}^{q}\left(R^{\prime \prime}, R\right)\right) \Rightarrow \operatorname{Ext}_{R}^{p+q}(M, R)
$$

has only one nonzero row, $q=1$, from which the result follows.

Lemma 4.5. If $\mathscr{A}$ is generic non-Boolean of rank $\ell \geq 4$ then there is a short exact sequence

$$
0 \longrightarrow \Omega_{0}^{p}(\mathscr{A})(-1) \stackrel{\alpha_{H}}{\longrightarrow} \Omega_{0}^{p}\left(\mathscr{A}^{\prime}\right) \longrightarrow \Omega_{0}^{p}\left(\mathscr{A}^{\prime \prime}\right) \longrightarrow 0
$$

for $0 \leq p \leq \ell-3$.

Proof. We first establish the case $p=1$. From Ziegler's presentation of $\Omega^{1}(\mathscr{A})$ of a generic arrangement [39, Cor. 7.7], we have $\operatorname{pdim}_{S} \Omega_{0}^{1}(\mathscr{A})=\operatorname{pdim}_{S} \Omega^{1}(\mathscr{A})=1$. So $\Omega_{0}^{1}(\mathscr{A})$ is 1 -spherical with zero-dimensional non-free locus. It follows that its dual, $D^{0}(\mathscr{A})$, is $(\ell-2)$-spherical, by Proposition 3.10. Since $\ell-2>1$, we have $\operatorname{Ext}_{R}^{1}\left(D^{0}(\mathscr{A}), R\right)=0$. By Lemma 4.4, we have

$$
\operatorname{Ext}_{R}^{1}\left(D^{0}\left(\mathscr{A}^{\prime \prime}\right), R\right)=\operatorname{Hom}_{R^{\prime \prime}}\left(D^{0}\left(\mathscr{A}^{\prime \prime}\right), R^{\prime \prime}\right)(1), \quad R^{\prime \prime}=R / R \alpha_{H} .
$$

Recall that $D_{\bullet}^{0}(\mathscr{A})$ and $\Omega_{0}^{\bullet}(\mathscr{A})$ are mutually $R$-dual by [9, Prop. 3.5]. Lemma 4.6 below gives exactness of (4.6) for $p=1$.

For general $p$, we proceed as follows. As both multiplication by $\alpha_{H}$ and restriction to $H$ commute with contraction against $\chi$, passing to the kernels of $\chi$ in (4.2) gives exactness of (4.6), except at the right module. 
Since $\mathscr{A}^{\prime}$ and $\mathscr{A}^{\prime \prime}$ are again generic, they are both free outside points and tame. So we have $\bigwedge^{p} \Omega_{0}^{1}\left(\mathscr{A}^{\prime}\right) \cong \Omega_{0}^{p}\left(\mathscr{A}^{\prime}\right)$ for $p \leq \ell-2$ and $\bigwedge^{p} \Omega_{0}^{1}\left(\mathscr{A}^{\prime \prime}\right) \cong \Omega_{0}^{p}\left(\mathscr{A}^{\prime \prime}\right)$ for $p \leq \ell-3$, by [9, Prop. 2.9]. Now by surjectivity in case $p=1$ and right-exactness of $\bigwedge^{p},(4.6)$ is exact on the right as well.

Lemma 4.6. The sequence (4.6) for $p=1$ is obtained by dualizing (4.3) and applying (4.5) for $M=D^{0}\left(\mathscr{A}^{\prime \prime}\right)$ and $q=0$.

Proof. Ignoring degrees and dropping 0-indices, we need to show that the restriction map $\Omega^{1}\left(\mathscr{A}^{\prime}\right) \longrightarrow \Omega^{1}\left(\mathscr{A}^{\prime \prime}\right)$ from $(4.2)$ coincides with the composition of the connecting homomorphism

$$
\operatorname{Hom}_{S}\left(D\left(\mathscr{A}^{\prime}\right), S\right) \longrightarrow \operatorname{Ext}_{S}^{1}\left(D\left(\mathscr{A}^{\prime \prime}\right), S\right) \cong \operatorname{Hom}_{S^{\prime \prime}}\left(D\left(\mathscr{A}^{\prime \prime}\right), S^{\prime \prime}\right),
$$

obtained from dualizing (4.1), with the isomorphism

$$
\operatorname{Ext}_{S}^{1}\left(D\left(\mathscr{A}^{\prime \prime}\right), S\right) \cong \operatorname{Hom}_{S^{\prime \prime}}\left(D\left(\mathscr{A}^{\prime \prime}\right), S^{\prime \prime}\right),
$$

obtained from (4.5) with $M=D\left(\mathscr{A}^{\prime \prime}\right)$ and $q=0$.

The claim is trivially true outside the arrangement $\mathscr{A}^{\prime \prime}$. The source and target of these two maps are naturally isomorphic and (reflexive, hence,) normal. Therefore it suffices to prove the statement locally at a generic point of $\mathscr{A}^{\prime \prime}$.

The arrangement $\mathscr{A}$ is generic; thus, in a generic point of $\mathscr{A}^{\prime \prime}, \mathscr{A}$ is the product of a Boolean 2-arrangement with $\mathbb{C}^{\ell-2}$. In particular, $D\left(\mathscr{A}^{\prime \prime}\right)_{x}, D\left(\mathscr{A}^{\prime}\right)_{x}$ and $D(\mathscr{A})_{x}$ are free in such a point. Then (4.1) is a free resolution of $D\left(\mathscr{A}^{\prime \prime}\right)_{x}$ and the claim becomes an exercise in homological algebra.

Abbreviate $A=D\left(\mathscr{A}^{\prime}\right)_{x}, B=D(\mathscr{A})_{x}$, and $C=D\left(\mathscr{A}^{\prime \prime}\right)_{x}$; then the complex (4.1) becomes

$$
0 \longrightarrow A \stackrel{\alpha}{\longrightarrow} B \longrightarrow C \longrightarrow 0
$$

and it has a free resolution

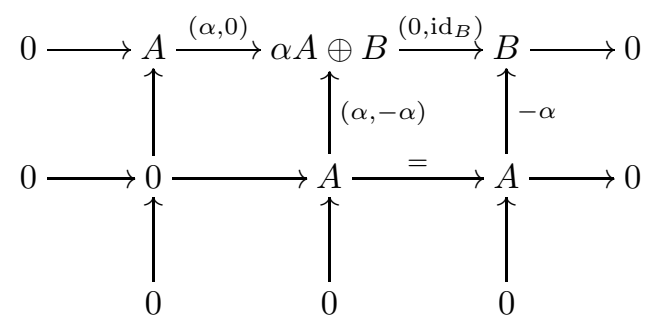

After dualizing, the connecting homomorphism $A^{\vee} \longrightarrow A^{\vee} / \alpha^{\vee} B^{\vee}$ shows up in the $E^{2}$-page of the spectral sequence of the double complex. It is induced by $\left(\alpha^{-1} \circ \alpha\right)^{\vee}=\mathrm{id}_{A^{\vee}}$, so it coincides with the natural restriction map.

Since our calculation does not depend on the choice of equations for the generic arrangement, let $\mathscr{A}_{n, \ell}$ denote a generic arrangement of $n$ hyperplanes with rank $\ell$.

The base case of our induction argument will be the following.

Lemma 4.7. For $\mathscr{A}=\mathscr{A}_{n, 3}$ with $n>3, \operatorname{Ext}_{S}^{1}\left(\Omega_{0}^{1}(\mathscr{A}), S\right)$ is an Artinian module with Hilbert series

$$
q_{n}(t)=\left((n-3) t^{-1}+(1-n)+(n-1) t^{n-3}+(3-n) t^{n-2}\right) /(1-t)^{3} .
$$


Proof. By [39, Cor. 7.7], one has a free resolution

$$
0 \longrightarrow S(-1)^{n-3} \longrightarrow S^{n-1} \longrightarrow \Omega_{0}^{1}(\mathscr{A}) \longrightarrow 0 .
$$

On the other hand, following [37], the module of derivations for the generic arrangement has a graded free resolution

$$
0 \longrightarrow S(2-n)^{n-3} \longrightarrow S(3-n)^{n-1} \longrightarrow D^{0}(\mathscr{A}) \longrightarrow 0,
$$

so $h\left(D^{0}(\mathscr{A}), t\right)=\left((n-1) t^{n-3}-(n-3) t^{n-2}\right) /(1-t)^{3}$. Now we dualize (4.7) to obtain

$$
0 \longleftarrow \operatorname{Ext}_{S}^{1}\left(\Omega_{0}^{1}(\mathscr{A}), S\right) \longleftarrow S^{n-3}(1) \longleftarrow S^{n-1} \longleftarrow D^{0}(\mathscr{A}) \longleftarrow 0,
$$

and take the Euler characteristic to compute the Hilbert series of $\operatorname{Ext}_{S}^{1}\left(\Omega_{0}^{1}(\mathscr{A}), S\right)$.

Remark 4.8. The previous result can be expressed more concisely in terms of a generating function. One can calculate that

$$
\begin{aligned}
\sum_{n \geq 4} q_{n}(t) s^{n} & =\frac{1}{(1-t)^{3}} \sum_{n \geq 4}\left((n-3) t^{-1}+(1-n)+(n-1) t^{n-3}+(3-n) t^{n-2}\right) s^{n} \\
& =\frac{1}{(1-t)^{3}}\left(\frac{s^{4} t^{-1}}{(1-s)^{2}}+\frac{(2 s-3) s^{4}}{(1-s)^{2}}+\frac{(3-2 s t)\left(s^{4} t\right)}{(1-s t)^{2}}-\frac{s^{4} t^{2}}{(1-s t)^{2}}\right) \\
& =t^{-1} \frac{s^{4}}{(1-s)^{2}(1-s t)^{2}} .
\end{aligned}
$$

In particular, by setting $t=1$ in the expression above, we obtain $s^{4} /(1-s)^{4}$, and we find that $\operatorname{Ext}_{S}^{1}\left(\Omega_{0}^{1}(\mathscr{A}), S\right)$ is a module of length equal to $\left(\begin{array}{c}n-1 \\ 3\end{array}\right)$.

Proof of Theorem 4.1. Suppose that the conclusion is known for all arrangements of fewer than $n$ hyperplanes. We prove the claim for $\mathscr{A}_{n, \ell}$ where $n>\ell$. We will assume $\ell \geq 4$, since the rank 3 case is covered by Lemma 4.7. By hypothesis, $\Omega_{0}^{p}\left(\mathscr{A}^{\prime}\right)$ and $\Omega_{0}^{p}\left(\mathscr{A}^{\prime \prime}\right)$ are $p$-spherical for $1 \leq p \leq \ell-3$. Now apply $\operatorname{Ext}_{R}^{\bullet}(-, R)$ to the short exact sequence (4.6). By Lemma 4.4, we may replace $\operatorname{Ext}_{R}^{q+1}\left(\Omega_{0}^{p}\left(\mathscr{A}^{\prime \prime}\right), R\right)$ by $\operatorname{Ext}_{R^{\prime \prime}}^{q}\left(\Omega_{0}^{p}\left(\mathscr{A}^{\prime \prime}\right), R^{\prime \prime}\right)$ in the long exact sequence. Then the $p$-spherical condition, together with the exactness of (4.3) for $i=0$, implies that the long exact sequence breaks up as

$$
0 \longrightarrow \operatorname{Ext}_{S}^{i}\left(\Omega_{0}^{p}\left(\mathscr{A}^{\prime}\right), S\right)(-1) \longrightarrow \operatorname{Ext}_{S}^{i}\left(\Omega_{0}^{p}(\mathscr{A}), S\right) \longrightarrow \operatorname{Ext}_{S^{\prime \prime}}^{i}\left(\Omega_{0}^{p}\left(\mathscr{A}^{\prime \prime}\right), S^{\prime \prime}\right) \longrightarrow 0
$$

for all $i \geq 0$ and for $0 \leq p \leq \ell-3$.

On the other hand, for $p=\ell-2$, note $p \geq 2$. Consider the long exact sequence obtained from the dual of (4.4). By Lemma 4.6, it begins with the sequence (4.6), which is exact from Lemma 4.5. It follows that $\operatorname{Ext}_{S}^{1}\left(\Omega_{0}^{\ell-2}(\mathscr{A}), S\right)=0$.

For $i \geq 2$, the assumption that $\Omega_{0}^{\ell-2}\left(\mathscr{A}^{\prime}\right)$ and $\Omega_{0}^{\ell-3}\left(\mathscr{A}^{\prime \prime}\right)$ are $(\ell-2)$-and $(\ell-3)$ spherical, respectively, gives a short exact sequence

$$
0 \rightarrow \operatorname{Ext}_{S^{\prime \prime}}^{i-1}\left(\Omega_{0}^{\ell-3}\left(\mathscr{A}^{\prime \prime}\right), S^{\prime \prime}\right)(1) \rightarrow \operatorname{Ext}_{S}^{i}\left(\Omega_{0}^{\ell-2}(\mathscr{A}), S\right) \rightarrow \operatorname{Ext}_{S}^{i}\left(\Omega_{0}^{\ell-2}\left(\mathscr{A}^{\prime}\right), S\right) \rightarrow 0 .
$$

It follows that $\Omega_{0}^{p}(\mathscr{A})$ is $p$-spherical for $1 \leq p \leq \ell-3$ by (4.8), and for $p=\ell-2$ by (4.9). In either case, the length of $\operatorname{Ext}_{S}^{p}\left(\Omega_{0}^{p}(\mathscr{A}), S\right)$ can be computed using the 
induction hypothesis and (4.8) or (4.9) to be

$$
\left(\begin{array}{c}
n-2 \\
\ell
\end{array}\right)+\left(\begin{array}{c}
n-2 \\
\ell-1
\end{array}\right)=\left(\begin{array}{c}
n-1 \\
\ell
\end{array}\right)
$$

If one repeats the argument, it is possible to obtain the Hilbert series of each $\operatorname{Ext}_{S}^{p}\left(\Omega_{0}^{p}(\mathscr{A}), S\right)$, instead of just its length. Given the number of parameters involved, we present this refinement to Theorem 4.1 separately.

Definition 4.9. Let $q(\ell, n, p ; t)$ denote the Hilbert series of $\operatorname{Ext}_{S}^{p}\left(\Omega_{0}^{p}\left(\mathscr{A}_{n, \ell}\right), S\right)$, for $3 \leq \ell \leq n$ and $1 \leq p \leq \ell-2$. Let $Q(\ell, p ; s, t)$ be the generating function

$$
Q(\ell, p ; s, t)=\sum_{n \geq 3} q(\ell, n, p ; t) s^{n},
$$

and write $Q(\ell, p)$ for short.

We saw, above, that

$$
Q(3,1)=s^{4} /\left(t(1-s)^{2}(1-s t)^{2}\right) .
$$

Lemma 4.10. The generating functions $Q(\ell, p)$ satisfy

$$
Q(\ell, p)= \begin{cases}\frac{s}{1-s t} Q(\ell-1, p) & \text { for } 1 \leq p \leq \ell-3 \\ \frac{s}{t(1-s)} Q(\ell-1, \ell-3) & \text { for } p=\ell-2 .\end{cases}
$$

Proof. The short exact sequence (4.8), for $i=p$, gives

$$
q(\ell, n, p ; t)=t q(\ell, n-1, p ; t)+q(\ell-1, n-1, p ; t)
$$

as long as $1 \leq p \leq \ell-3$. Writing the sum of both sides gives the equation $Q(\ell, p ; s, t)=s t Q(\ell, p ; s, t)+s Q(\ell-1, p ; s, t)$, from which we obtain the formula above. The case $p=\ell-2$ is similar, using (4.9) instead.

By applying this result recursively, beginning with (4.10), we obtain the expression:

Lemma 4.11. For all $\ell \geq 3$ and $1 \leq p \leq \ell-2$,

$$
Q(\ell, p ; s, t)=t^{-p} \frac{s^{\ell+1}}{(1-s)^{p+1}(1-s t)^{\ell-p}} .
$$

Continuing further, let

$$
P(p ; s, t, u)=\sum_{\ell \geq p+2} Q(\ell, p ; s, t) u^{\ell} .
$$

Then the geometric series formula, using (4.11), simplifies to the following:

$$
P(p ; s, t, u)=\frac{s^{3} u^{2}}{(1-s)(1-s t)(1-s(t+u))}\left(\frac{s u}{t(1-s)}\right)^{p},
$$

for $p \geq 1$. Finally, we can form a four-variable generating function

$$
T(s, t, u, v)=\sum_{p \geq 1} P(p ; s, t, u) v^{p} .
$$

By construction,

$$
T(s, t, u, v)=\sum_{n, \ell, p} h\left(\operatorname{Ext}_{S}^{p}\left(\Omega_{0}^{p}\left(\mathscr{A}_{n, \ell}\right), S\right), t\right) s^{n} u^{\ell} v^{p} .
$$


Since each module is Artinian, $T \in \mathbb{Z}\left[t, t^{-1}\right][[s, u, v]]$ (and, in particular, our rational function calculations do lead to a valid expression for a formal power series). By summing the expression (4.12), we obtain the following:

Corollary 4.12. The Hilbert series of the module $\operatorname{Ext}_{S}^{p}\left(\Omega_{0}^{p}\left(\mathscr{A}_{n, \ell}\right), S\right)$ is the coefficient of $s^{n} u^{\ell} v^{p}$ in

$$
T(s, t, u, v)=\frac{s^{4} u^{3} v}{(1-s)(1-s t)(1-s t-s u)(t-s t-s u v)},
$$

for all $1 \leq p \leq \ell-2 \leq n-2$.

Acknowledgments. We would like to express our gratitude to the American Institute of Mathematics in Palo Alto for its support and hospitality during our three SQuaRE meetings, out of which this work grew.

\section{REFERENCES}

1. A. Borel, P.-P. Grivel, B. Kaup, A. Haefliger, B. Malgrange, and F. Ehlers, Algebraic Dmodules, Perspectives in Mathematics, vol. 2, Academic Press Inc., Boston, MA, 1987. MR882000 (89g:32014) 1.1

2. Egbert Brieskorn, Singular elements of semi-simple algebraic groups, Actes du Congrès International des Mathématiciens (Nice, 1970), Tome 2, Gauthier-Villars, Paris, 1971, pp. 279-284. MR0437798 (55 \#10720) 1

3. James W. Bruce, Functions on discriminants, J. London Math. Soc. (2) 30 (1984), no. 3, 551-567. MR810963 (87e:58028) 1

4. Francisco J. Calderón-Moreno, Logarithmic differential operators and logarithmic de Rham complexes relative to a free divisor, Ann. Sci. École Norm. Sup. (4) 32 (1999), no. 5, 701-714. MR1710757 (2000g:32010) 2.10

5. Francisco J. Calderón Moreno, David Mond, Luis Narváez Macarro, and Francisco J. Castro Jiménez, Logarithmic cohomology of the complement of a plane curve, Comment. Math. Helv. 77 (2002), no. 1, 24-38. MR1898392 (2003e:32047) 2.3

6. Francisco J. Castro-Jiménez, Luis Narváez-Macarro, and David Mond, Cohomology of the complement of a free divisor, Trans. Amer. Math. Soc. 348 (1996), no. 8, 3037-3049. MR1363009 (96k:32072) 2.3

7. D. Cohen, G. Denham, M. Falk, and A. Varchenko, Critical points and resonance of hyperplane arrangements, Canad. J. Math. 63 (2011), no. 5, 1038-1057. MR2866070 1.3, 3.3

8. Ignacio de Gregorio, David Mond, and Christian Sevenheck, Linear free divisors and Frobenius manifolds, Compos. Math. 145 (2009), no. 5, 1305-1350. MR2551998 1

9. Graham Denham and Mathias Schulze, Complexes, duality and Chern classes of logarithmic forms along hyperplane arrangements, Arrangements of hyperplanes-Sapporo 2009, Adv. Stud. Pure Math., vol. 62, Math. Soc. Japan, Tokyo, 2012, pp. 27-57. MR2933791 1.1, 1.2, $1.2,3.1,3.1,3.1,4,4,4$

10. Paul H. Edelman and Victor Reiner, A counterexample to Orlik's conjecture, Proc. Amer. Math. Soc. 118 (1993), no. 3, 927-929. MR1134624 (93i:52021) 1

11. David Eisenbud, Craig Huneke, and Wolmer Vasconcelos, Direct methods for primary decomposition, Invent. Math. 110 (1992), no. 2, 207-235. MR1185582 (93j:13032) 2.1, 2.1

12. Michel Granger, David Mond, Alicia Nieto-Reyes, and Mathias Schulze, Linear free divisors and the global logarithmic comparison theorem, Ann. Inst. Fourier (Grenoble) 59 (2009), no. 2, 811-850. MR2521436 1

13. Michel Granger, David Mond, and Mathias Schulze, Free divisors in prehomogeneous vector spaces, Proc. Lond. Math. Soc. (3) 102 (2011), no. 5, 923-950. MR2795728 1, 2.3

14. Michel Granger and Mathias Schulze, On the formal structure of logarithmic vector fields, Compos. Math. 142 (2006), no. 3, 765-778. MR2231201 (2007e:32037) 2.3, 2.3, 2.3

15. - On the symmetry of b-functions of linear free divisors, Publ. RIMS 46 (2010), no. 3, 479-506. MR2760735 (2011k:14014) 1, 2.3

16. Karsten Lebelt, Zur homologischen Dimension äusserer Potenzen von Moduln, Arch. Math. (Basel) 26 (1975), no. 6, 595-601. MR0396534 (53 \#397) 3.1, 3.1 
17. _ Freie Auflösungen äusserer Potenzen, Manuscripta Math. 21 (1977), no. 4, 341-355. MR0450253 (56 \#8549) 3.1

18. E. J. N. Looijenga, Isolated singular points on complete intersections, London Mathematical Society Lecture Note Series, vol. 77, Cambridge University Press, Cambridge, 1984. MR747303 (86a:32021) 1

19. Marguerite Mangeney, Christian Peskine, and Lucien Szpiro, Anneaux de Gorenstein, et torsion en algèbre commutative, Séminaire d'Algèbre Commutative dirigé par Pierre Samuel, 1966/67. Texte rédigé, d'après des exposés de Maurice Auslander, Marquerite Mangeney, Christian Peskine et Lucien Szpiro. École Normale Supérieure de Jeunes Filles, Secrétariat mathématique, Paris, 1967. MR0225844 (37 \#1435) 3.1

20. Hideyuki Matsumura, Commutative ring theory, second ed., Cambridge Studies in Advanced Mathematics, vol. 8, Cambridge University Press, Cambridge, 1989, Translated from the Japanese by M. Reid. MR1011461 (90i:13001) 2.1

21. David Mond and Mathias Schulze, Adjoint divisors and free divisors, arXiv.org math.AG (2010), no. 1001.1095, Submitted. 1

22. Mircea Mustaţă and Henry K. Schenck, The module of logarithmic p-forms of a locally free arrangement, J. Algebra 241 (2001), no. 2, 699-719. MR1843320 (2002c:32047) 2.9, 3.1

23. Peter Orlik and Hiroaki Terao, Arrangements of hyperplanes, Grundlehren der Mathematischen Wissenschaften [Fundamental Principles of Mathematical Sciences], vol. 300, SpringerVerlag, Berlin, 1992. MR1217488 (94e:52014) 1.3, 1.6, 4

24. Jan-Erik Roos, Bidualité et structure des foncteurs dérivés de $\lim _{\longrightarrow}$ dans la catégorie des modules sur un anneau régulier, C. R. Acad. Sci. Paris $\overrightarrow{\mathbf{5 4}}(1962), 1556-1558$. MR0136640 (25 \#106b) 2.3

25. Lauren L. Rose and Hiroaki Terao, A free resolution of the module of logarithmic forms of a generic arrangement, J. Algebra 136 (1991), no. 2, 376-400. MR1089305 (93h:32048) 1.3

26. Kyoji Saito, Quasihomogene isolierte Singularitäten von Hyperflächen, Invent. Math. 14 (1971), 123-142. MR029469945 \#3767 2.16

27. - Theory of logarithmic differential forms and logarithmic vector fields, J. Fac. Sci. Univ. Tokyo Sect. IA Math. 27 (1980), no. 2, 265-291. MR58645083h:32023 1, 2.3, 2.11, 2.3, $2.3,2.3,3.1$

28. Christian Sevenheck, Bernstein polynomials and spectral numbers for linear free divisors, Ann. Inst. Fourier (Grenoble) 61 (2011), no. 1, 379-400. MR2828135 1

29. Aron Simis, Differential idealizers and algebraic free divisors, Commutative algebra, Lect. Notes Pure Appl. Math., vol. 244, Chapman \& Hall/CRC, Boca Raton, FL, 2006, pp. 211226. MR2184799 (2006k:13055) 1

30. Peter Slodowy, Simple singularities and simple algebraic groups, Lecture Notes in Mathematics, vol. 815, Springer, Berlin, 1980. MR584445 (82g:14037) 1

31. Louis Solomon and Hiroaki Terao, A formula for the characteristic polynomial of an arrangement, Adv. in Math. 64 (1987), no. 3, 305-325. MR88863188m:32022 2.2

32. Hiroaki Terao, Free arrangements of hyperplanes and unitary reflection groups, Proc. Japan Acad. Ser. A Math. Sci. 56 (1980), no. 8, 389-392. MR596011 (82e:32018a) 1

33. , Discriminant of a holomorphic map and logarithmic vector fields, J. Fac. Sci. Univ. Tokyo Sect. IA Math. 30 (1983), no. 2, 379-391. MR722502 (85d:32027) 1

34. Duco van Straten, A note on the discriminant of a space curve, Manuscripta Math. 87 (1995), no. 2, 167-177. MR1334939 (96e:32032) 1

35. Jonathan Wiens, The module of derivations for an arrangement of subspaces, Pacific J. Math. 198 (2001), no. 2, 501-512. MR1835521 (2002d:14090) 4

36. Jonathan Wiens and Sergey Yuzvinsky, De Rham cohomology of logarithmic forms on arrangements of hyperplanes, Trans. Amer. Math. Soc. 349 (1997), no. 4, 1653-1662. MR1407505 (97h:52013) 1.3

37. Sergey Yuzvinsky, A free resolution of the module of derivations for generic arrangements, J. Algebra 136 (1991), no. 2, 432-438. MR1089307 (92b:52026) 4

38. V. M. Zakalyukin, Reconstructions of fronts and caustics depending on a parameter, and versality of mappings, Current problems in mathematics, Vol. 22, Itogi Nauki i Tekhniki, Akad. Nauk SSSR Vsesoyuz. Inst. Nauchn. i Tekhn. Inform., Moscow, 1983, pp. 56-93. MR735440 (85h:58029) 1

39. Günter M. Ziegler, Combinatorial construction of logarithmic differential forms, Adv. Math. 76 (1989), no. 1, 116-154. MR1004488 (90j:32016) 5, 4, 4, 4 
Department of Mathematics, University of Western Ontario, London, Ontario, Canada N6A 5B7

E-mail address: gdenham@uwo.ca

URL: http://www.math.uwo.ca/ gdenham

Department of Mathematics, University of Illinois, Urbana, IL 61801

E-mail address: schenck@math.uiuc.edu

URL: http://www. math.uiuc.edu/ schenck

Department of Mathematics, University of Kaiserslautern, 67663 Kaiserslautern, GERMANY

E-mail address: mschulze@mathematik.uni-kl.de

URL: http://www.mathematik.uni-kl.de/ mschulze

Department of Mathematics, United States Naval Academy, Annapolis, MD 21402

E-mail address: wakefiel@usna.edu

URL: http://www. usna.edu/Users/math/wakefiel

Department of Mathematics, Purdue University, West Lafayette, IN 47907

E-mail address: walther@math.purdue.edu

URL: http://www . math. purdue. edu/ walther 